\title{
Microparticles Formation of Ganoderma Iucídum Extract by Electrospraying Method
}

\author{
Siti Machmudah *,1 \\ Dwi Setyorini ${ }^{1}$ \\ Sugeng Winardi ${ }^{1}$ \\ Wahyudiono $^{2}$ \\ Hideki Kanda ${ }^{2}$ \\ Motonobu Goto ${ }^{2}$ \\ 1 Department of Chemical Engineering, Faculty of Industrial Technology, Institut Teknologi \\ Sepuluh Nopember, Surabaya 60111, Indonesia \\ 2 Department of Materials Process Engineering, Graduate School of Engineering, Nagoya \\ University, Nagoya 464-8603, Japan \\ *e-mail: machmudah@chem-eng.its.ac.id
}

In this work, Ganoderma lucidum (G. lucidum) extract was produced in microparticles form by electrospraying. G. lucidum was extracted hydrothermally at temperature of $160^{\circ} \mathrm{C}$ and pressure of $7 \mathrm{MPa}$. The extract solution was subsequently mixed with $6 \%$ of Polyvinyl pyrrolidone (PVP) and formed into microparticles by electrospraying process. The electrospraying was carried out at applied voltage of 12,14 , and $16 \mathrm{kV}$, and the distance between syringe tip and electrospun collector of 8,10 , and $12 \mathrm{~cm}$. The microparticles formed was analyzed using scanning electron microscope (SEM), fourier-transform infrared (FTIR) spectroscopy, and UV-Vis spectrofotometer. The antioxidant efficiency of particles was also analyzed by 1,1-diphenyl-2-picrylhydrazyl (DPPH) assay. Based on the SEM analysis, the G. lucidum extract (GLE) - PVP spherical particles were formed by electrospraying. The finer fibres were clearly formed with the increasing applied voltage. The results showed that applied voltage and distance of tip to electrospun collector significantly influence the antioxidant efficiency and the diameter size of particles. The antioxidant efficiency increased with the rising applied voltage and gap of tip to electrospun collector, while the particle diameter decreased with the rising applied voltage and gap of tip to electrospun collector due to fast mass transfer and evaporation. The largest antioxidant efficiency of particles was $0.377 / \mathrm{min}$ obtained at $16 \mathrm{kV}$ and $12 \mathrm{~cm}$. It indicated that electrospraying is an effective process to produce pharmaceutical compounds in powder form.

Keywords: Ganoderma lucidum, electrospraying, microparticles, hydrothermal extraction

\section{INTRODUCTION}

Ganoderma lucidum (G. lucidum), known as Reishi in Japan and lingzhi in
China, was considered as a medicinal fungus for over 2000 years. It contains various phytochemical compounds including triterpenoids and flavonoids. The 
particular utilizations and the health advantages of $G$. lucidum encompass hepatoprotection, the immune system modulation, bacteriostasis, and restraint of blood glucose levels (Bishop et al. 2015). G. lucidum is not only applied in a medical field but also utilized as cosmetics, health supplement, and nutraceutical. Various utilizations in traditional medicine field have attracted the researchers attention to conduct clinical and preclinical trials and therapeutical applications such as antioxidant (Krishna et al. 2016), anticancer (Jin et al. 2012), antinociceptive (Sheena et al. 2005), immunomodulator (Ishimoto et al. 2017), antiarthritic (Pan et al. 2017), hypoglycaemic (Pan et al. 2014), cardioprotective (Rajasekaran and Kalaimagal 2012), anti-allergic (Ji et al. 2007), anti-inflammatory (Cai et al. 2017), carcinostatic (Sliva 2003), antiangiogenic (Chen et al. 2017)], antiosteoporotic (Elhassaneen et al. 2016), proapoptotic (Gill and Kumar 2016), antiviral (Wang et al. 2004), and anti-HIV (Akbar and Yam 2011).

Various methods have been introduced to generate the particles including supercritical anti-solvent, precipitation of solvent-nonsolvent, coacervation, spray drying, polymerization via sol-gel-based, and oil-in-water or water-in-oil emulsion. Nevertheless, these methods possess some drawbacks, i.e. costly apparatus, the product degradation owing to the heating during the process, the generation of particle size is relatively big, and the remaining organic solvent in the product. (Paximada et al. 2017).

Electrospraying was applied to generate drug particles, inorganic particles, entrapped the active ingredient particles, and drug-delivery polymer particles (Gomez-Estaca et al. 2012). Electrospraying is a one-stage processing method to produce the particles via electrical forces. The operating conditions that govern the particle size and its distribution are concentration of polymer solution, tip to electrospun collector distance, flow rate, electric voltage, and environmental humidity (Gaona-Sanchez et al. 2016). Recently, electrospraying was employed to generate microparticles of $G$. lucidum loaded sodium alginate in order to preserve the G. lucidum structure. Zhao et al. (2016) prepared and characterized the G. lucidum spore (GLS)-alginate microbead by electrospraying with varying the applied voltage and drying processes. They reported that the releasing velocity of GLS encapsulation was affected by the solution $\mathrm{pH}$ and the drying process. The particles produced had the size of 600 to $2000 \mu \mathrm{m}$. Yao et al. (2017) employed the electrospraying for generating G. lucidum polysaccharide (GLP) loaded sodium alginate microparticles with the size ranging $255-355 \mu \mathrm{m}$. They varied the collection environment temperature $(\sim 25$ to $50^{\circ} \mathrm{C}$ ) and obtained that GLP/Naalginate micro-particles were modified with diverse surface morphologies (crinkled and porous) without resulting in the change in material chemical composition. Zhu et al. (2019) extracted GLP by traditional solvent extraction (TSE) and ultrasound-assisted extraction (UAE), and subsequently encapsulated with polyvinyl alcohol (PVA) to form nanofibers with the size ranging from 390 to $750 \mathrm{~nm}$ by electrospraying. They reported that GLP concentration influenced the maximum 
fibrous composite films tensile/strain strength, contact angle of water, and mean diameter of fiber. Until now, application of electrospraying on the GLP-polyvinyl pyrrolidone (PVP) microparticles has not been reported yet in the literatures.

Therefore, in this work G. lucidum was extracted hydrothermally at temperature $160^{\circ} \mathrm{C}$ and pressure $7 \mathrm{MPa}$ based on the optimum condition of preliminary findings. G. lucidum extract (GLE) was subsequently mixed with PVP to generate microparticles by electrospraying. The effects of the applied voltage and the tip to electrospun collector gap on the particles morphology and characterization were investigated.

\section{MATERIALS AND METHODS}

\section{Materials}

Ganoderma lucidum was bought from the local market in Semarang, central Java, Indonesia. Polyvinyl pyrrolidone (PVP) and 1,1-diphenyl-2-picrylhydrazyl (DPPH) were obtained from Sigma Aldrich. Methanol and ethanol were supplied by Merck.

\section{Hydrothermal Extraction}

In this research, hydrothermal extraction was conducted in a semi-batch extractor. The primary device of hydrothermal extraction consisted of extractor $(10 \mathrm{ml}$ in volume; Thar Design Inc., USA), back pressure regulator (BPR; AKICO, Japan), high pressure pump (200 LC Pump, Perkin Elmer, Germany), and heater (Memmert UN 55). There were removable threaded covers included stainless-steel filters $(0.1-1 \mu \mathrm{m})$ in both side of extractor. For the extraction, initially, $1 \mathrm{~g}$ of raw material was filled into the extractor. The glass beads were loaded in the both sides of extractor to avoid channeling. When the heater was equipped with the extractor, the HPLC pump flowed water at $1 \mathrm{ml} \mathrm{min}{ }^{-1}$ flow rate into the extractor. And then, back pressure regulator (BPR) was applied to control the pressure according to the pressure gauge that monitors the pressure. Water temperature was increased using a heater to reach the desired temperature. The temperature in inlet and outlet extractor were measured by thermocouple $T_{1}$ and $T_{2}$ respectively, to monitor the temperature inside the extractor related to the operating temperature. Next the solution of extract was quenched by using heat exchanger, then pass through the filter, and collected in the sample bin. The extraction was conducted at temperature of $160^{\circ} \mathrm{C}$ and pressure of $7 \mathrm{MPa}$ for 3 hours. The apparatus scheme of hydrothermal extraction device is described in Fig. 1.

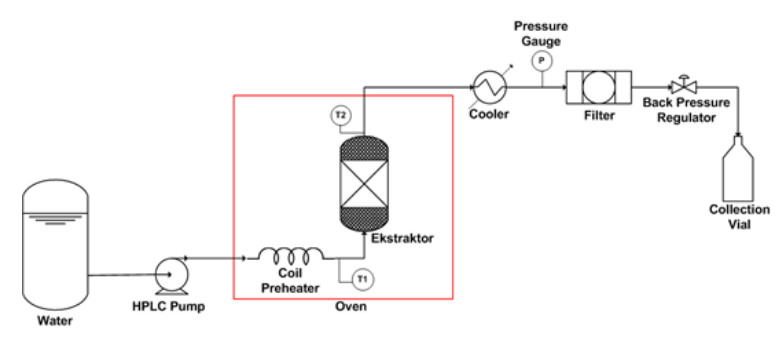

Fig. 1: Apparatus scheme of hydrothermal extraction device

\section{Electrospraying}

The primary apparatus of electrospraying was consisted of high voltage supply power (high voltage power supply model HGR30-20N, Japan), syringe pump (KD Scientific, USA), and an electrospun collector made of aluminum 
foil. The schematic diagram of electrospraying apparatus is shown in Fig. 2. In the electrospraying process, firstly, the precursor consisted of solution GLE and PVP with the concentration of $6 \% \mathrm{w} / \mathrm{v}$ was prepared sonically. The conductivity of precursor solution was measured by a conductometer to ensure that the precursor has enough charges. The humidity was monitored at that time. Next, the precursor solution was put in the syringe pump to spray. The tip of syringe was charged with a high voltage generated by the power supply. The applied voltage was varied at 12,14 , and $16 \mathrm{kV}$. The resulting particles would stick to the electrospun collector that has a different charge with the tip of syringe. The electrospraying was carried out at a distance between tips and electrospun collector of 8,10 , and $12 \mathrm{~cm}$ for 4 hours.

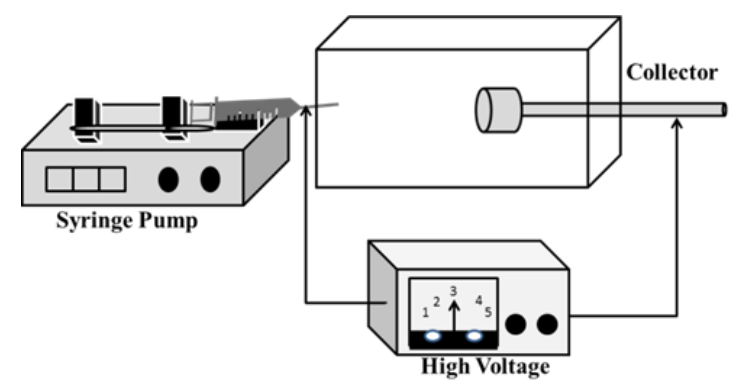

Fig. 2: Schematic diagram of electrospraying apparatus

\section{Analytical Method}

The morphology of particles formed was analyzed by scanning electron microscopy (SEM; JEOL JSM-6390LV, Tokyo, Japan). The particles sizes were determined by ImageJ software based on the SEM images; and the standard deviation was calculated by statistical analysis using ANOVA. The structure of particles was determined by a Spectrum Two FTIR spectrophotometer (Perkin Elmer Ltd., Buckinghamshire, England) with the wavenumber ranging from 4000 to 400 $\mathrm{cm}^{-1}$. The antioxidant activity of particles was also determined by DPPH assay with the Genesys 10 UV-Vis Scanning Spectrophotometer (Thermo Fisher Scientific, Waltham, MA) at wavelength of $516 \mathrm{~nm}$ for analyzing the absorbance of remaining methanolic DPPH solution reacted with the particles. The antioxidant activity was expressed as antioxidant efficiency.

\section{RESULTS AND DISCUSSION}

The influence of applied voltage on the GLE-PVP particles shape was investigated at PVP concentration of $6 \%$ and $12 \mathrm{~cm}$ tip to electrospun collector gap. Fig. 3 shows the SEM images of particles obtained at various applied voltages. The spherical particles were formed for all applied voltages. The fine fibers were also formed with the increased in applied voltage. The diameter size of particles slightly decreased from $1.181 \pm 0.543 \mu \mathrm{m}$, $0.985 \pm 0.494 \mu \mathrm{m}$, and $0.943 \pm 0.446 \mu \mathrm{m}$ with increasing applied voltage for $12 \mathrm{kV}, 14 \mathrm{kV}$, and $16 \mathrm{kV}$, respectively. Theoretically, the increase in electric voltage increases the current via the solution cone and may influence the mechanism of jet break-up. At the surface of a jet, the ratio of stress which is determined by the stress of surface tension and the stress of normal electric, is the key parameter to specify the electrified jet break up mode. The instabilities of varicose are the jet breakup owing to the instabilities of 
axisymmetric at a value of the low-stress ratio. In this case, a single disperse droplet might be produced from the small quantity of a secondary droplet. Nevertheless, when the higher voltage was introduced, it increases the current via the solution jet and accordingly improves the charge of the surface and the ratio of stress. The solution jet starts to thin, and the instabilities of lateral may promote the jet break-up above a value of the stress ratio threshold that can produce the smaller droplet.

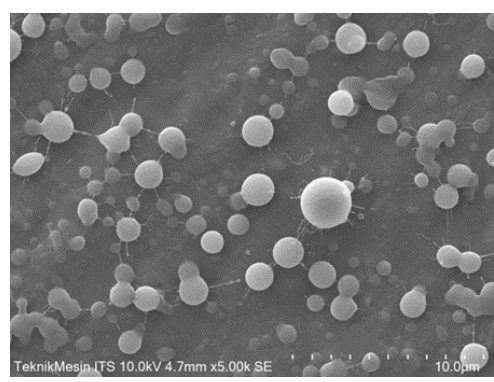

(a)

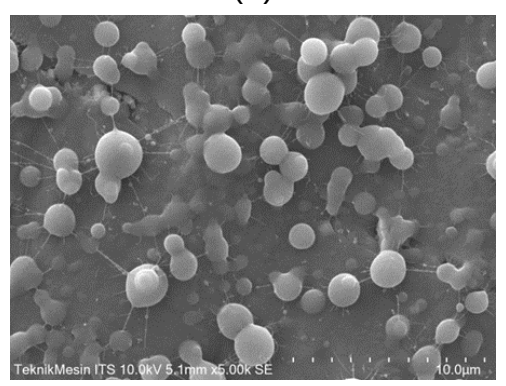

(b)

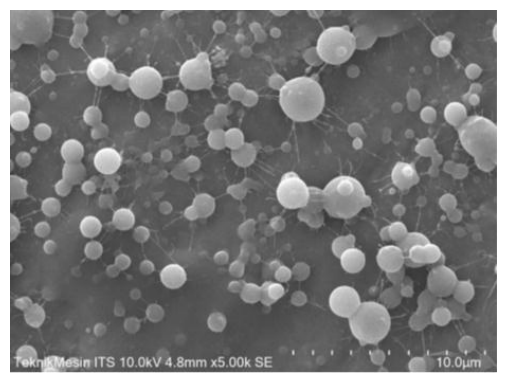

(c)

Fig. 3: SEM images of particles produced by electrospraying at various applied voltages. (a) $12 \mathrm{kV}$; (b) 14 $\mathrm{kV}$; (c) $16 \mathrm{kV}$.
The SEM images of particles formed by electrospraying at various tip to electrospun collector distances and constant applied voltage of $14 \mathrm{kV}$ and PVP concentration of $6 \%$ are shown in Fig. 4 . It was observed that the particles in wet condition with agglomeration were obtained at $8 \mathrm{~cm}$ gap (low distance). At this distance, the diameter particles were $1.429 \pm 0.564 \mu \mathrm{m}$. Yet, when the gap expanded to 10 and $12 \mathrm{~cm}$, the smaller spherical shaped particles with diameter size of $0.991 \pm 0.460 \mu \mathrm{m}$ and $0.985 \pm 0.494$ $\mu \mathrm{m}$, respectively, were formed. This phenomenon can be explained as follows. When the gap between the tip to the electrospun collector is very short, the polymer-solvent might not have sufficient time to remove entirely via evaporation prior to achieving the electrospun collector. As a result, the particles aggregation occurs. Conversely, the long distance between the tip to the electrospun collector might cause in a decrease in the strength of an electric field, however, it could expand the evaporation time for the polymer-solvent. Hence, the electrospun might be lost to the surroundings when it transfers from the tip to the electrospun collector. If an apt distance was set in an electrospraying system, the polymersolvent possess sufficient time to remove completely; this drives to a reduce in the particles coalescence and rise in the efficiency of electrospun collection (Chhouk et al. 2018). In this study, the GLEPVP particles were formed uniformly at long tip to electrospun collector distance.

In this work, the antioxidant activity of GLE-PVP particles was expressed as antioxidant efficiency (AE) that describes 
the effectivity of certain concentration of a component against reactive oxygen/nitrogen species (ROS/RON). The particles were dissolved in water and added into DPPH methanolic solution to measure the decreasing absorbance of initial DPPH solution. The effect of electrospraying condition on the antioxidant efficiency of particles is listed on Tabel 1.

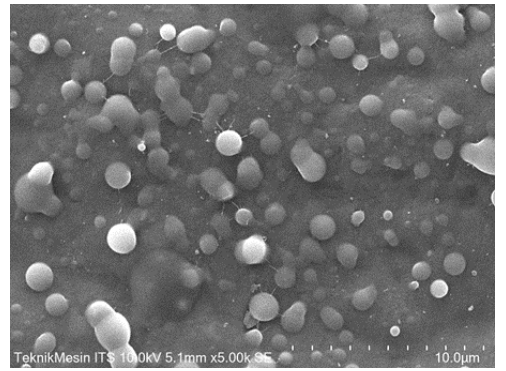

(a)

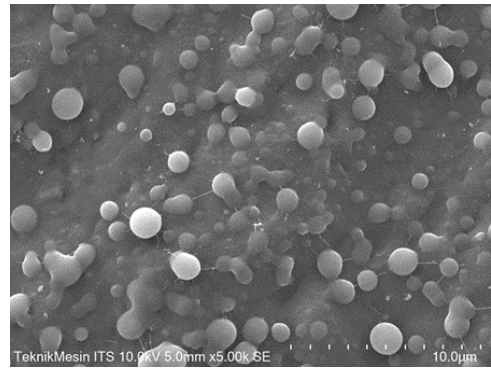

(b)

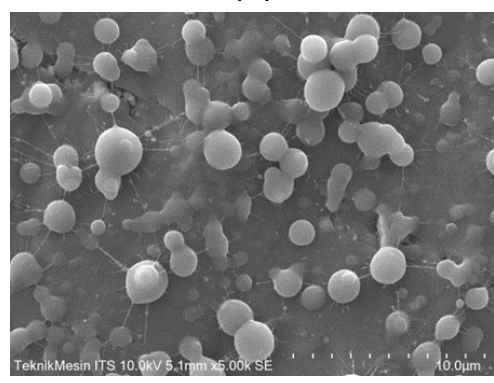

(c)

Fig. 4: SEM images of particles produced by electrospraying at various tip to electrospun collector gaps. (a) 8 cm; (b) $10 \mathrm{~cm}$; (c) $12 \mathrm{~cm}$.

As illustrated in Table 1, in general the increasing applied voltage caused the increasing antioxidant efficiency. As mentioned before, the increasing applied voltage also caused the decreasing diameter particles. It indicated that the active components in the particles are distributed uniformly in the particles. Moreover, in the same number of particles may contain more active components compared to the particles obtained at smaller applied voltage. This result also indicated that electrospraying does not change the nature of the active compounds.

Table 1. Antioxidant efficiency of GLE-PVP particles at various electrospinning conditions

\begin{tabular}{cccc}
\hline $\begin{array}{c}\text { PVP } \\
\begin{array}{c}\text { Concentration } \\
\text { (\%) }\end{array}\end{array}$ & $\begin{array}{c}\text { Applied } \\
\text { Voltage } \\
(\mathbf{k V})\end{array}$ & $\begin{array}{c}\text { Tip to } \\
\text { electrospun } \\
\text { collector } \\
\text { distance } \\
\text { (cm) }\end{array}$ & $\begin{array}{c}\text { AE } \\
\left(\mathbf{m i n}^{-\mathbf{1}}\right)\end{array}$ \\
\hline 6 & 12 & 8 & 0.099 \\
\hline 6 & 14 & 8 & 0.136 \\
\hline 6 & 16 & 8 & 0.196 \\
6 & 12 & 10 & 0.119 \\
\hline 6 & 14 & 10 & 0.233 \\
6 & 16 & 10 & 0.308 \\
\hline 6 & 12 & 12 & 0.159 \\
6 & 14 & 12 & 0.247 \\
\hline 6 & 16 & 12 & 0.377 \\
\hline
\end{tabular}

In order to observe the structure of components that composed the GLE-PVP particles, the particles were analyzed by FTIR. Fig. 5 shows the FTIR chromatogram of GLE-PVP particles produced at various applied voltages. The particles were composed of similar component structures. The particles contained functional group of antioxidant compounds, such as flavonoids and phenolic compounds. The sharp peaks of $\mathrm{C}-\mathrm{O}$ in the range of 1710 $\mathrm{cm}^{-1}, \mathrm{C}=\mathrm{C}$ in the range of $1630 \mathrm{~cm}^{-1}$, and 
$\mathrm{C}=\mathrm{O}$ in the range of $1155 \mathrm{~cm}^{-1}$ are appeared as typical functional group of flavonoids (Mot et al., 2011). Moreover, the particles contained active compounds of ganoderic acids with functional group of amides in the range of $1242 \mathrm{~cm}^{-1}$. Functional group of $\beta$-D-glucan in the range of $895 \mathrm{~cm}^{-1}$ is also appeared as the most active compound in G. lucidum.

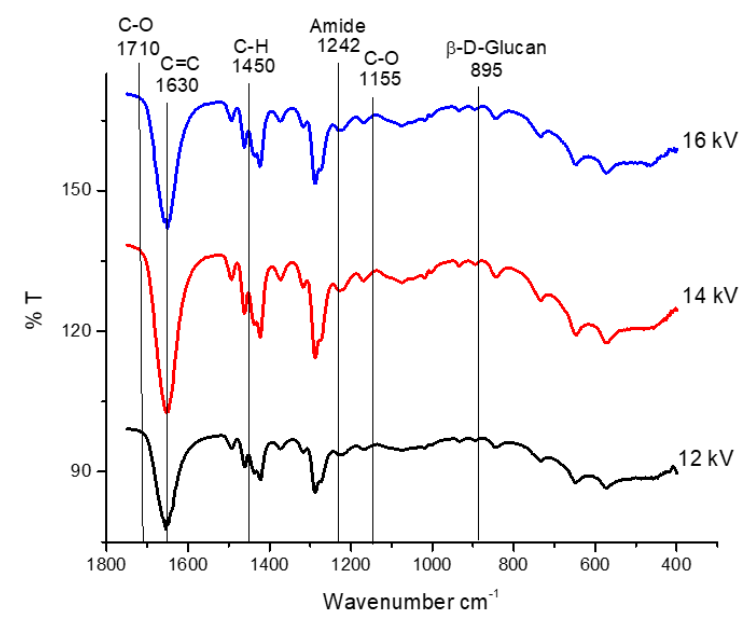

Fig. 5: FTIR chromatogram of GLE-PVP particles produced at various applied voltages.

\section{CONCLUSIONS}

Ganoderma lucidum extract (GLE) particles have been produced by electrospraying at various applied voltages and tip to electrospun collector distances. The spherical particles were formed at all operating conditions. As increasing applied voltage, the finer fibers were also formed. The diameter particles increased with increasing applied voltage and tip to electrospun collector gap but decreased with the PVP concentration increased. The diameter size of particles ranged from 0.943 to $1.429 \mu \mathrm{m}$. The GLE-PVP particles contained antioxidant compounds. The antioxidant efficiency of particles was affected by electrospraying operating condition. However, the structure of component functional group does not change with the operating condition. The best condition of the electrospraying process was found at $16 \mathrm{kV}$ applied voltage and $12 \mathrm{~cm}$ the tip to electrospun collector gap. This work indicated that electrospraying method is capable to be an alternative method to produce microparticles.

\section{ACKNOWLEDGEMENT}

Authors wish to acknowledge the financial support from Directorate General of Science, Technology and Higher Education, Ministry of Research, Technology and Higher Education of the Republic of Indonesia through a research grant "World Class Research" under contract No. 128/SP2H/LT/DRPM/2019 and No. 710/PKS/ITS/2019.

\section{REFERENCES}

1. Akbar, R., Yam, W. K. (2011). "Interaction of ganoderic acid on HIV related target: molecular docking studies," Bioinformation, 7, 413-417.

2. Bishop, K. S., Kao, C. H. J., Xu, Y., Glucina, M. P., Paterson, R. R. M., Ferguson, L. R. (2015). From 2000 years of Ganoderma lucidum to recent developments in nutraceuticals," Phytochemistry, 114, 56-65.

3. Cai, Q., Li, Y., Pei, G. (2017). "Polysaccharides from Ganoderma lucidum attenuate microglia- 
mediated neuroinflammation and modulate microglial phagocytosis and behavioural response," J. Neuroinflammation 14, 63-70.

4. Chen, S., Yong, T., Zhang, Y., Su, J., Jiao, C., Xie, Y. (2017). "Anti-tumor and anti-angiogenic ergosterols from Ganoderma lucidum," Front. Chem., 5, 85-70.

5. Chhouk, K., Wahyudiono, Kanda, H., Goto, M. (2018). "Micronization for Enhancement of Curcumin Dissolution via Electrospraying Technique," ChemEngineering, 2, 6066.

6. Elhassaneen, Y. A., Ragab, S. S., Salman, M. S. (2016). "The Potential Effects of Reishi Mushroom (Ganoderma lucidum) Consumption on Bone Health Indices and Serum Minerals Profile Disorders Induced by $\mathrm{CCl} 4$ on Rats," Pyrex J. Medicinal Pant Res., 2, 001-007.

7. Gaona-Sanchez, V. A., CalderonDominguez, G., Morales-Sanchez, E., Chanona-Perez, J. J., Arzate-Vazquez, I., Terres-Rojas, E. (2016). "Pectinbased films produced by electrospraying," J. Appl. Polym. Sci., https://doi.org/10.1002/app.43779.

8. Gill, B. S. and Kumar, S. (2016) "Evaluating anti-oxidant potential of ganoderic acid A in STAT 3 pathway in prostate cancer," Mol. Biol. Rep., 43, 1411-1422.

9. Gomez-Estaca, J., Balaguer, M. P., Gavara, R., Hernandez-Muooz, P. (2012). "Formation of zein nanoparticles by electrohydrodynamic atomization: Effect of the main processing variables and suitability for encapsulating the food coloring and active ingredient curcumin," Food Hydrocoll., 28, 82-91.

10. Ishimoto, Y., Ishibashi, K. -I., Yamanaka, D., Adachi, Y., Ito, H., Igami, K., Miyazaki, T., Ohno, N. (2017). "Enhanced release of immunostimulating B-1, 3-Glucan by autodigestion of the lingzhi medicinal mushroom, ganoderma lingzhi (Agaricomycetes)," Int. J. Med. Mushrooms, 19, 1-16.

11. Ji, Z., Tang, Q., Zhang, J., Yang, Y., Jia, W., Pan, Y. (2007). "Immunomodulation of RAW264. 7 macrophages by GLIS, a proteopolysaccharide from Ganoderma lucidum," J. Ethnopharmacol., 112, 445-450.

12. Jin, X., Beguerie, J. R., Sze, D. M.-Y., Chan, G. (2012). "Ganoderma lucidum (Reishi mushroom) for cancer treatment," Cochrane Database Syst. Rev., 6.

13. Krishna, K. V., Karuppuraj, V., Perumal, K. (2016). "Antioxidant activity and Folic acid content in indigenous isolates of Ganoderma lucidum," Asian J. Pharm. Anal., 6, 213-215.

14. Pan, D., Zhang, D., Wu, J., Chen, C., Xu, Z., Yang, H., Zhou, P. (2014). "A novel proteoglycan from Ganoderma lucidum fruiting bodies protects kidney function and ameliorates diabetic nephropathy via its antioxidant activity in C57BL/6 db/db mice," Food Chem. Toxicol., 63, 111-118.

15. Pan, X., Lopez-Olivo, M. A., Song, J., Pratt, G., Suarez-Almazor, M. E. 
(2017). "Systematic review of the methodological quality of controlled trials evaluating Chinese herbal medicine in patients with rheumatoid arthritis," BMJ Open, 7, e013242. doi: 10.1136/bmjopen2016-013242.

16. Paximada, P., Echegoyen, Y., Koutinas, A. A., Mandala, L. G., Lagaron, J. M. (2017). "Encapsulation of hydrophilic and lipophilized catechin into nanoparticles through emulsion electrospraying," Food Hydrocoll., 64, 123-132.

17. Rajasekaran, M. and Kalaimagal, C. (2012). "Cardioprotective effect of a medicinal mushroom, Ganoderma lucidum against adriamycin induced toxicity," Int. J. Pharmacol., 8, 252258.

18. Sudheesh, N. P., Ajith, T. A., Ramnath V., Janardhanan K. K. (2010). "Therapeutic potential of Ganoderma lucidum (Fr.) P. Karst. against the declined antioxidant status in the mitochondria of postmitotic tissues of aged mice.," Clin Nutr., 29, 406-412.

19. Sliva, D. (2003) "Ganoderma lucidum (Reishi) in cancer treatment," Integr. Cancer Ther., 2, 358-364.

20. Wang, Y., Zhang, L., Li, Y., Hou, X., Zeng, F. (2004). "Correlation of structure to antitumor activities of five derivatives of a B-glucan from Poria cocos sclerotium," Carbohydr. Res., 339, 2567-2574.

21. Yao, Z. -C., Jin, L. -J., Ahmad, Z., Huang, J., Chang, M. -W., Li, J. -S. (2017). "Ganoderma lucidum polysaccharide loaded sodium alginate micro-particles prepared via electrospraying in controlled deposition environments," Int. J. Pharmaceutics, 524, 148-158.

22. Zhao, D., Li, J. -S., Suen, W., Chang, M.-W., Huang, J. (2016). "Preparation and characterization of Ganoderma lucidum spores-loaded alginate microspheres by electrospraying," Mat. Sci. Eng. C, 62, 835-842.

23. Zhu, L. -F., Chen, X., Ahmad, Z., Li, J. S., Chang, M. -W., (2019). "Engineering of Ganoderma lucidum polysaccharide loaded polyvinyl alcohol nanofibers for biopharmaceutical delivery," J. Drug Deliv. Sci. Technol., 50, 2. 